\title{
Increasing the range of ultra wideband radars based on antenna arrays
}

\author{
Boris Lagovsky ${ }^{1, *}$, Alexander Samokhin ${ }^{1}$, and Malay Tripathy ${ }^{2}$ \\ ${ }^{1}$ Russian Technological University (MIREA), 78, Vernadsky av., Moscow, Russia \\ ${ }^{2}$ Amity University, Noida - 201313, Uttar Pradesh, India
}

\begin{abstract}
Analytical and numerical solutions are presented for increasing the range of ultra wideband radars based on antenna arrays by optimizing their characteristics. It is shown that the shape of UWB pulses significantly affects the maximum value of the reflected signal. Solutions of variational problems of searching for the optimal shape of probing UWB pulses are investigated. Optimization of the form allows to obtain good results when using even approximate data on the dispersion dependences of the radar cross-section. The use of new efficient processing algorithms for UWB signals that do not have direct narrow-band analogs is substantiated.
\end{abstract}

\section{Introduction}

A large number of research centers in the United States, Russia, China, France, Japan and other countries are actively working in the field of the theory of UWB signals, UWB technologies [1-3]. The most developed area of application for UWB locators is the detection and observation of objects at short distances. In recent years, UWB-signals have become increasingly considered for use in classical radar. To date, several types of remote sensing UWB radars have been developed.

UWB-location potentially provides: - reduction of time for detection and recognition of targets; - high resolution in range and accuracy of ranging; - a significant reduction in the effect of passive interference; - the possibility of selecting targets due to high spatial resolution.

The application of UWB radar can significantly expand with an increase in their range. Progress in the development of more powerful generators allows you to count on it. However, a significant increase in the range can be achieved without increasing the energy of the radio channel, only by optimizing the characteristics of UWB radars.

\section{Formulation of the problem}

Consider the problem of increasing the range of UWB radars with a fixed energy of the emitted pulses. In other words, it is required to find the form or, in an alternative formulation, the spectral composition of the UWB-generated pulse, which ensures the maximum radar range.

\footnotetext{
*Corresponding author: Robertlag@yandex.ru
} 
The tactical and technical characteristics of the radar when using UWB pulses are significantly different from the characteristics when using traditional narrow-band signals. The spectrum and temporal structure of UWB pulses emitted by an antenna array (AA) may differ significantly from the spectrum and temporal structure of the generated pulses due to the dispersion properties of the directional pattern (DP) and the mutual influence of the AA elements [4-7].

When calculating and modeling the received signals, in addition to the dispersion properties of the AA, the dispersion properties of the RCS should also be taken into account.

The radar target will be described using a complex reflection coefficient $R$. The function $R$ depends on the frequency of the probe signal $\omega$ and the angular position of the target $(\alpha, \beta)$ relative to the radar.

$$
R(\alpha, \beta, \omega)=\sqrt{\sigma(\alpha, \beta, \omega)} \exp (i \varphi(\alpha, \beta, \omega)),
$$

where $\sigma(\alpha, \beta, \omega)$ is the Radar Cross-Section (RCS) normalized to the maximum, $\varphi(\alpha, \beta, \omega)$ is the phase-response characteristic of reflection.

In the future, for brevity, we assume that the target is located in the direction $(0,0)$ and denote the introduced values $\sigma(\omega), \varphi(\omega)$ and $R(\omega)$.

Let the function $U_{r}(t)$ describe the time dependence of the received pulse. One of the most important pulse detection parameters is its maximum (peak) value $U_{M}$ of the signal.

The solution of the problem is to determine the shape of the UWB pulse $U(t)$, including the values of the highest and lowest frequencies of the spectrum $\omega_{\max }$ and $\omega_{\min }$, which provide the maximum value of $U_{M}$ under two additional conditions.

1) The energy of all the studied pulse types must be the same, i.e.

$$
\int_{0}^{\tau} U^{2}(t) d t=\int_{\omega_{\min }}^{\omega_{\max }} V(\omega) V^{*}(\omega) d \omega=\text { const }
$$

where $\tau$ is the pulse duration, $V(\omega)$ is the complex spectrum of the generated pulse to be determined, $V^{*}(\omega)$ is the complex conjugate function. This condition allows you to correctly compare the obtained solutions.

2) The width of the received DP should not exceed the specified width $\theta_{0,5}$. This condition provides angular measurements with the required accuracy.

Usually, in addition to detecting a target, it is necessary to measure its coordinates. The second additional condition shows the specifics of using UWB signals. The width of the DP is dependent on the signal spectrum.

To simplify the expressions obtained in the future, we will consider the shape of the RP at each frequency to be symmetrical in angle with respect to the direction to the maximum. Then DP depends only on the angle of deviation from the maximum. The maximum value of a signal received from this direction can be represented as:

$$
U_{M}(\theta)==\underbrace{\max }_{t}\left(\int_{\omega_{\min }}^{\omega_{\max }} V(\omega)|F(\theta, \omega)|^{2} R(\omega) \exp (i \omega t) d \omega\right)
$$

where $F(\theta, \omega)$ is the DP.

From (3), in particular, it follows that the width of the DP $\theta_{0,5}$, when using UWB pulses is different when emitting and receiving signals. The width and shape of the DP depend not only on the dispersion characteristics of AA, but also on the width and shape of the 
spectrum of the UWB pulses and the frequency dependence of the reflection coefficient of the target $R(\omega)$.

Using (3), we represent the second additional condition of the problem in the form:

$$
\frac{\sqrt{2}}{2} \underbrace{\max }_{t}\left(\int_{\omega_{\min }}^{\omega_{\max }} V(\omega)|F(0, \omega)|^{2} R(\omega) \exp (i \omega t) d \omega\right)=\underbrace{\max }_{t}\left(\int_{\omega_{\min }}^{\omega_{\max }} V(\omega)|F(\theta, \omega)|^{2} R(\omega) \exp (i \omega t) d \omega\right)
$$

When solving the reflection characteristics of the radar target $R(\omega)$ is considered known. This involves the use of advance information about the target. In the absence of such information, any mathematical model of the frequency dependence of the RCS should be used.

The mathematical formulation of the problem: find the spectrum of the generated signal $V(\omega)$,providing at $\theta=0$ the maximum of (3). There are given: frequency dependencies of the DP $F(\omega, \theta)$, characteristics of the reflection of the target in the form of a complex function $R(\omega$, additional conditions (2) and (4).

\section{Research methods}

We will divide the solution of the problem into two stages. At the first stage, an algorithm is constructed for searching the spectrum of the generated signal $V(\omega)$ for given fixed values of $\omega_{\max }$ and $\omega_{\min }$. At the second stage, the values of the highest and lowest frequencies, the spectrum of the UWB pulses $V(\omega)$ and the corresponding maximum possible value $U_{\mathrm{M}}(0)$ are found.

The first stage is reduced to solving a variation problem. Entering the uncertain Lagrange multipliers $\lambda_{1}$ and $\lambda_{2}$, we arrive at a solution in the form:

$$
\begin{gathered}
V_{o p t}(\omega)=\frac{1}{\lambda_{2}} R^{*}(\omega)\left\{F(0, \omega)^{2^{*}}-\lambda_{1}\left[F(0, \omega)^{2^{*}}-\sqrt{2} F\left(\theta_{0}, \omega\right)^{2^{*}} \exp (-i \omega \Delta t)\right]\right\} \\
\lambda_{1}=\frac{0.5 \int_{\omega_{\min }}^{\omega_{\max }} R(\omega) R^{*}(\omega)\left\{F(0, \omega)^{2^{*}} \exp \left(-i \omega t_{0}\right) \Phi(\omega)+F(0, \omega)^{2} \exp \left(i \omega t_{0}\right) \Phi^{*}(\omega)\right\} d \omega}{\int_{\omega_{\min }}^{\omega_{\max }} R(\omega) R^{*}(\omega) \Phi(\omega) \Phi^{*}(\omega) d \omega} \\
\Phi(\omega)=\frac{\sqrt{2}}{2} F(0, \omega)^{2} \exp \left(i \omega t_{0}\right)-F\left(\theta_{0}, \omega\right)^{2} \exp \left(i \omega t_{1}\right)
\end{gathered}
$$

where $t_{0}$ and $t_{1}$ are the arrival times of the maxima of the UWB pulses received from the directions $\theta=0$ and $\theta=\theta_{0}, \Delta t=t_{0}-t_{1}$. The normalizing factor $\lambda_{2}$ is found from the additional condition (2).

At the second stage of the solution, for each pair of $\omega_{\max }$ and $\omega_{\min }$, the value of the received signal $U_{M}(0)$ (Eq.(1)) is found. Comparing all obtained values $U_{M}(0)$, we find the maximum value, the corresponding boundary frequencies $\omega_{\max }, \omega_{\min }$ and the optimal spectrum of the generated UWB signal $V_{\text {opt }}(\omega)(5)$.

As an example, consider the detection of a target with a reflection coefficient $R(\omega)$ close to the frequency dependence of the ultrawideband radio absorbing coating. The maximum $R(\omega)$ falls on the low-frequency region, when the electrical coating thickness is much less than the wavelength. At higher frequencies, the value of $R(\omega)$ decreases rapidly. 
The maximum allowed width of the antenna array of the antenna array was $\theta_{0.5}=3^{\circ}$. The frequency $f$ and the pulse duration $\tau$ are expressed in relative units, so that the condition: $f \tau=1$. That is, if the frequency $f$ is given in $\mathrm{GHz}$, then $\tau$ is in nanoseconds.

Calculations are presented in dimensionless quantities. For $f_{0}=1$, the value of the frequency at which $\theta_{0.5}=3^{\circ}$. At this frequency, the antenna size is $\sim 20 \lambda_{0}$.

To evaluate the effectiveness of the optimization carried $K_{\mathrm{W}}$ factor has been introduced. It shows how many times the signal-to-noise (SN) ratio in the received signal has increased in comparison with the average value for narrowband signals in the used frequency range. We consider noise as "white".

Figure 1 shows the dependence of the factor $K_{\mathrm{W}}$ on the low-frequency boundary of the spectrum $f_{\min }$ found for various fixed values of the high-frequency boundary: $f_{\min }=0,2 f_{0}$ (1); $f_{\min }=0,25 f_{0}(2) ; f_{\min }=0,275 f_{0}(3)$.

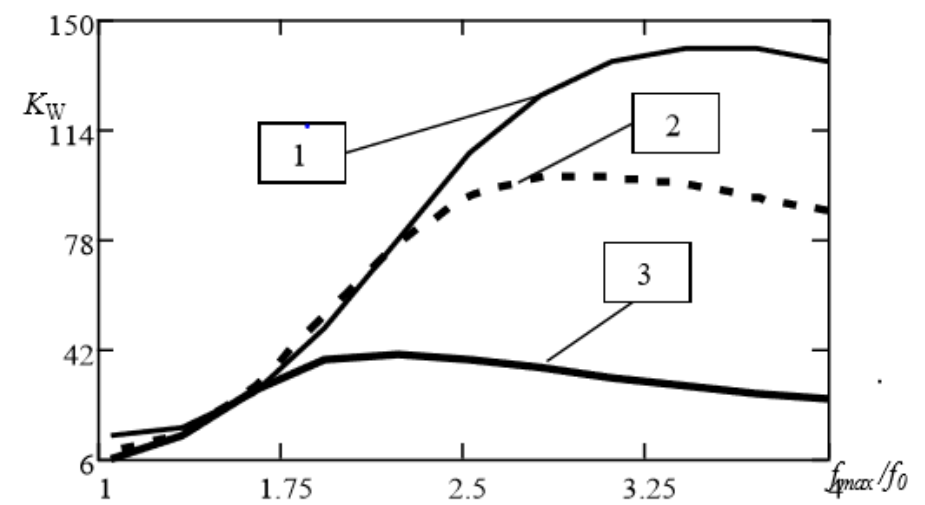

Fig. 1. The dependence of the factor $K_{\mathrm{W}}$ on the low-frequency boundary.

Figure 2 shows the dependence of $K_{\mathrm{W}}$ on the high-frequency boundary $f_{\max }$ for various values of the low-frequency boundary: $f_{\max }=4 f_{0}(1) ; f_{\max }=2,82 f_{0}(2) ; f_{\max }=2,23 f_{0}(3)$

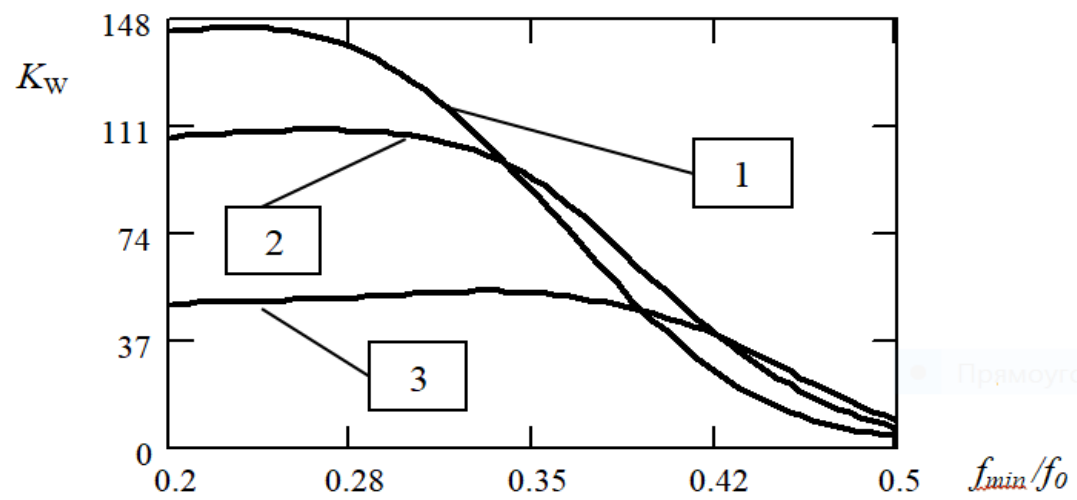

Fig. 2. The dependence of $K_{\mathrm{W}}$ on the high-frequency boundary.

From the obtained results, it follows that the SNR for optimal UWB signals is much larger than when using narrow-band signals. The maximum $K_{\mathrm{W}}$ was 144 , and was achieved with a bandwidth of $0.2 f_{0} \div 3.7 f_{0}$. This corresponds to an increase in the range of the radar by almost 4 times.

The use of optimal or quasi-optimal filtering allows you to further increase $K_{\mathrm{W}}$. The maximum $K_{\mathrm{W}}$ value was increased to 530 , i.e. the range of the system has increased by 5-6 times. 
In practice, the values of the function $R(\omega)$, especially its phase characteristics, can be given only approximately. The mathematical model was used to study the statistical dependence of the obtained $K_{\mathrm{W}}$ on the accuracy of setting the target reflection coefficient as compared to the true $R(\omega)$.

Figure 3 shows the results in the form of a decrease in the achieved $K_{\mathrm{W}}$ compared to the maximum possible $K_{\max }$ due to the root-mean-square error $\delta^{2}$ in specifying the function $R(\omega)$. Two types of errors are shown: in setting only the phase-frequency characteristic of the target reflection (1) and in setting only the amplitude-frequency characteristic (2).

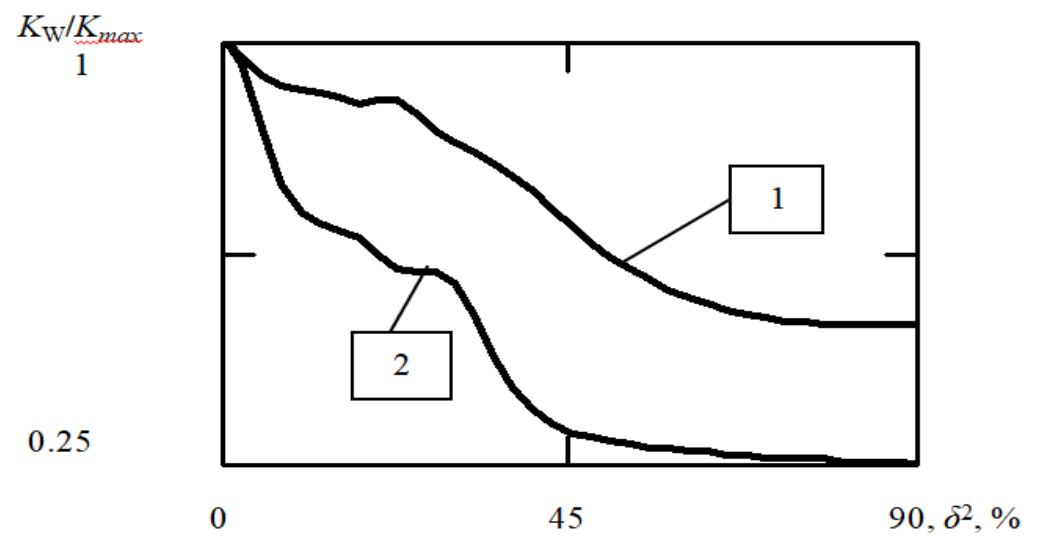

Fig. 3. $K_{\mathrm{W}}$ dependence on errors in specifying the target reflection characteristics.

The obtained simulation results show that the solutions found are stable. The $K_{\mathrm{W}}$ factor turns out to be noticeably more sensitive to amplitude errors than to phase errors. The average statistical reduction in $K_{\mathrm{W}}$ values, even with large errors of $50-90 \%$, is no more than 2-3 times compared to the maximum possible.

Thus, the optimization of the spectral composition (or shape) of UWB signals allows to obtain good results when using even approximate data on the dispersion dependence of the RCS.

\section{Formation of UWB pulses in the receiver of signals}

UWB generation of controlled spectrum pulses is a complex problem. It is not technically implemented yet.

The method of simplified control of the UWB pulse spectrum is proposed. It consists in using a packet of UWB pulses with different, preferably non-intersecting, spectra of individual pulses. The spectrum of each pulse is only part of the spectrum of the synthesized UWB pulse.

The receiving device carries out the accumulation of signals. The result is a single ultrawideband pulse with the required width of the spectrum.

Pulse summation can be performed with different weighting factors, which allows to vary the shape of the spectrum. Let a packet of $N$ pulses $U_{n}(t)$ with a pulse period $T$ was formed:

$$
U(t)=\sum_{n=1}^{N} c_{n} U_{n}(t-T \cdot n)
$$

Without loss of generality, we introduce the normalization of individual pulses with non-intersecting spectra in a pack and all the packs of UWB pulses: 


$$
\int_{-\infty}^{\infty} U_{n}(t)^{2} d t=1, \quad \int_{-\infty}^{\infty} U(t)^{2} d t=\sum_{n=1}^{N} c_{n}{ }^{2}=P=\text { const }
$$

We pose the problem of finding the optimal weight coefficients $c_{\mathrm{n}}$, which provide the maximum possible useful signal in the receiver. Set the number of pulses in a packet $N$, the frequency dependences of the DP $F(\omega, \theta)$, the spectra of each of the pulses $V_{n}(\omega)$. The additional condition ( 9 ) is introduced. In addition, the width of the DP should not exceed the specified value of $\theta_{0.5}$.

Introducing the spectra of each pulse $V_{\mathrm{n}}(\omega)$, we obtain the spectrum of the emitted pulse packet:

$$
V_{e}(\omega, \theta)=F(\omega, \theta) \sum_{n=1}^{N} c_{n} V_{n}(\omega)
$$

At the output of the receiving device after the accumulation of the signal, we obtain the spectrum of the final UWB pulse in the form:

$$
V_{r}(\omega, \theta, \varphi)=R(\omega) F^{2}(\omega, \theta, \varphi) \sum_{n=1}^{N} c_{n} V_{n}(\omega)
$$

By varying the coefficients $c_{\mathrm{n}}$, in accordance with Eq.(10) and Eq.(11), one can obtain different values of $U_{\max }$.

The solution of the problem is reduced to solving a system of linear algebraic equations with constraints. The numerical solution is carried out by standard methods and does not cause difficulties $[5,8,9]$.

As an example, we will give a solution to the problem already described earlier of detecting a target with a given reflection coefficient $R(\omega)$.

Formed pack of three pulses, the width of the spectrum of each of which is $1 / 3 \Delta f$, where $\Delta f$ is the width of the spectrum of the synthesized UWB pulse. The shapes of the spectra of individual pulses are given. In this example, each pulse is selected with uniform filling spectra bandwidth.

Figure 4 shows the time dependences of the emitted pulses of a pack. Dotted and thin curves are the first and third pulses. The bold curve is the total pulse of the whole packet in the receiver with the same $c_{n}$ without taking into account the dispersion properties of the reflection of the radar target.

The summation allowed us to create a 1 nanosecond pulse in the UWB receiver. The beamwidth was $8^{\circ}$ for the first pulse, and $1.6^{\circ}$ for the second. These values of the width of the DN are obtained with the weight coefficients $c_{n}(1,0,0)$ and $(0,0,1)$. With equal weights, the beamwidth was $6^{\circ}$, which turned out to be greater than the specified maximum allowable value of $3^{\circ}$.

Solving the optimization problem (9-11) for a target with a given dispersion type of the reflection coefficient $R(\omega)$ allowed us to find the values of the weight coefficients $c_{\mathrm{n}}$, which provide the highest possible SNR for a given beamwidth. The values of the found optimal coefficients $c_{\mathrm{n}}$ were $0.197 ; 0.632 ; 0.779$.

The maximum value of the UWB pulse with the found optimal coefficients $c_{\mathrm{n}}$ was slightly less than with identical coefficients. However, the beamwidth at the same time decreased by half and reached the specified value.

An increase in the number of pulses with non-intersecting spectra when allows to increase the maximum values of the total signal at reception. So, for the considered example, the maximum value of the total signal for a pack of 5 pulses is increased by $20 \%$ compared with a pack of 3 pulses. 


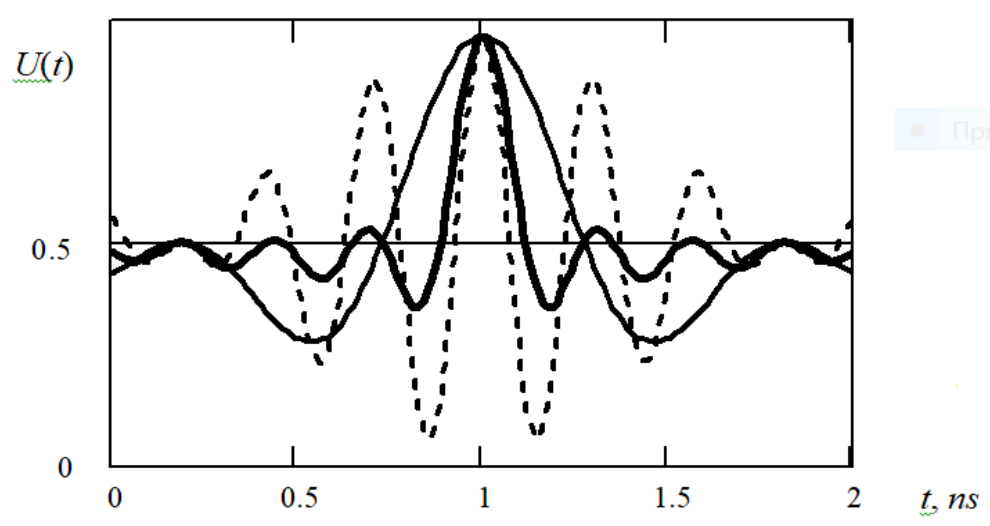

Fig. 4. The shape of the emitted and received pulses.

A further increase in the number of pulses significantly less affects the magnitude of the maximum.

Thus, the radiation of a pulse packet with non-intersecting spectra makes it possible, during reception, to generate the optimal UWB pulse necessary for detecting and measuring target coordinates with the required accuracy.

\section{Conclusions}

Analytical studies and the results of numerical experiments on a mathematical model show that the temporal structure (or spectrum) of UWB signals undergoes significant changes during emission, reflection and reception. As a result, the shape of the used UWB pulses significantly affects the maximum value of the reflected signal.

The problem of optimizing the spectral composition of UWB pulses on the basis of complete or partial data on the reflecting properties of the targets has been posed and solved. It is shown that it is possible to significantly increase the radar range by adapting the spectral composition of UWB pulses to the reflection characteristics of the target. Optimization of UWB signals allows to obtain good results when using even approximate data on the dispersion dependences of the RCS.

The reported study was partially supported by RFBR, research project No. 18-57-45001.

Authors are thankful to DST, New Delhi for the financial support (INT/RUS/RFBR/P-341) provided for the present work.

\section{References}

1. James D. Taylor, Ultra-wideband Radar Technology (CRC Press Boca Raton, London, New Work 2000)

2. G. Holami, H. Mehrpourbernety and B. Zakeri, UWB Phased Array Antennas for High Resolution Radars, Proc. of the 2013 International Symposium on Electromagnetic Theory, pp. 532-535 (2013)

3. B.A. Lagovsky, A.B. Samokhin, Y.V. Shestopalov, Increasing accuracy of angular measurements using UWB signals, 2017 11th European Conf. on Antennas and Propagation (EUCAP), Paris, pp. 1083 - 1086 (2017)

4. A.B. Samokhin, Y.V. Shestopalov, Pulse Characteristics of Antenna Array Radiating UWB Signals, Proc. of the 10th European Conf. on Antennas and Propagation (EuCAP), Davos, Switzerland, pp. 2479-2482 ( 2016) 
5. B.A. Lagovsky, A.G. Chikina, Shape optimization of UWB pulses, Progress In Electromagnetics Research Symposium (PIERS), pp. 475 - 478 (2017)

6. B.A. Lagovsky, A.B. Samokhin, A.S. Samokhina, Image restoration of the multiple target by smart antenna array radiating UWB signals, Proc. of Electromagnetics in Advanced Applications (ICEAA), Torino, Italy, pp.363 - 365 (2015)

7. B.A. Lagovsky, A.B. Samokhin, Superresolution in signal processing using a priori information, Inter. Conf. Electromagnetics in Advanced Applications (ICEAA), IEEE Conf. Publications, Verona, Italy, pp. 779 - 783 (2017)

8. B.A. Lagovsky, A.B. Samokhin, Y.V. Shestopalov, Increasing the Range of UWB Radars, Proc. 2018 12th European Conf. on Antennas and Propagation (EUCAP), UK, Ser. IET Conference Publications, v. CP741 (2018)

9. A.V. Smirnov, Method of simultaneous optimization of radio devices performance in frequency and time domains, Russian technological journal, v. 6, No. 6, pp. 13-27 (2018) 